\title{
Understanding of Meaning as a Communication Problem*
}

\author{
Alexei Cherniak \\ Faculty of Humanities and Social Sciences \\ Peoples' Friendship University of Russia \\ 6 Miklukho-Maklaya Str. \\ Moscow, Russia \\ E-mail: abishot2100@yandex.ru
}

\begin{abstract}
Our standard idea of communication presupposes that communication is based on the understanding of what is said or written, what other people mean by certain words and actions and etc. As representatives of different cultures speaking different languages so those who belong to the same culture and speak the same language need to be capable, as it seems, to make the words they hear or read meaningful in certain way in order to obtain understanding. But what exactly should be done depends on what is understood as meaning? There are different concepts of linguistic as well as communicative meaning. In this article, the conditions which have to be satisfied in order to obtain understanding of such widely discussed types of meaning as content, sense and denotation are investigated. It is shown that in neither case can understanding be literally assessing what other people say, think, presuppose, and so on.
\end{abstract}

Keywords-communication; understanding; meaning; denotation; content; sense; mental

\section{INTRODUCTION}

How can people of different cultures understand each other? By this question, different problems may be explicated. One set of these questions concerns the possibility of intercultural dialogue, where one problem is to reduce misunderstandings and conflicts generated by cultural differences by finding a common ground, and another is to create conditions in which any point of view provided by a particular culture could be expressed and taken into account by representatives of other cultures. Another set embraces rather linguistic than political problems. People belonging to different cultures often speak different languages; and, in communication with each other, they have to rely on translations of each other's utterances. But can these translations be proper, given the cultural differences? If the translation of the utterance $u$ made in a particular language $\mathrm{L}$ into the sentence of another language L' can only provide the information about what would $u$ say if it was made by the speaker of L', then what reason do we have to call that type of achievement understanding of what $u$ says?

The latter question is about meanings of words, and how

*The publication has been prepared with the support of the "RUDN University Program 5-100". they are connected with understanding. The same question may be asked about situations where people use or seem to use the same language. For if in communication its participants mean different things by the same words, how can they understand each other by assigning meanings to these words? We may say that if I utter the phrase "snow is white", meaning that grass is green, the one who knows English may still in a sense understand what I've said: at least, he/she may understand what the words I've used mean, and this is the proposition that snow is white. Whether that was what I've really said independently on what I thought I was saying, given that I was speaking English, is the matter of further discussion. However, when we call for understanding, we often mean understanding of what the speaker tried to say by using certain words in certain order. ${ }^{1}$

It seems that, when communicators share common concepts and beliefs, the understanding of meanings of words used in communication is not a big problem; it is easier to achieve in that case. Nevertheless, some philosophers claim that even when there is no significant conceptual disagreement between communicators, the judgments about meanings of the utterances produced within this communication, as well as about beliefs, intentions etc., which they express, cannot be verified [1]. At first glance, these seem to be two different problems. For we definitely cannot observe what's going on in one's head and, therefore, may assign beliefs and other attitudes to other people only with some probability reflecting mainly how similar the speaker is to the interpreter according to the past experience. On the other hand, it seems that we can say with much more certainty what words pronounced by others tell us if 1) we know the language of the utterance, and 2) meanings of the uttered words are not determined by beliefs, intentions etc. of their utterers. In a minimalist sense, any utterance means what may be read from its linguistic components by the competent speaker of the language of the utterance. It may mean much more but having this sort of meaning may serve as a minimal condition of successful understanding. We can plausibly assume that most linguistic expressions of particular languages have certain salient standard meanings commonly associated with them by their competent users. 
Thus, by assigning such meanings to expressions which we hear or read we may achieve some minimal degree of understanding of what was said. As certain type of result, understanding is often identified with correspondence of meanings, ${ }^{2}$ the one intended or meant by the speaker or writer and another assigned or read by the hearer or perceiver. If the hearer reads the words in such a way that they mean for him/her the same as for their utterer (when he/she uttered them), there is the correspondence of the kind considered. Then, if both assign to the same words appeared in the same order the same conventional meanings, there seems to be no problem in gaining such correspondence. In situations when communicators' conceptual backgrounds are different, we might assign some degree of understanding (lesser than maximum) according to how much correspondence of meanings was obtained. Still there is a problem with meaning, such that may undermine the possibility of understanding even in the minimal sense just considered.

\section{ASPECTS THAT DIFFERENT TYPES OF MEANING REQUIRE FROM UNDERSTANDING}

What we primarily achieve in understanding is something mental, whereas meanings are not necessarily mental. We may talk about meaning in different senses; but as semantic feature which words (at least when uttered or written) and mental states may have meaning is often recognized as a medium between words, utterances, and thoughts and our minds where we get interpretation and understanding. Moreover, some kinds of meaning by reference to which many philosophers of language and linguists explain many communicative phenomena are identified with external ${ }^{3}$ things and relations between them. Even when we say in our ordinary manner that we can understand other peoples' words, texts, and thoughts, we often presuppose that we can understand what these words, texts or thoughts are about. Part of meaning in this semantic sense may be being a mental reaction of understanding (or alike) to the perception of a sign, but there has to be another part: what is understood or otherwise grasped by the mind. Another frequently used term for some kinds of meaning is "content". Content may be recognized as something essentially subjective, and it may be presumed that understanding of the meaning of the utterance consists in reconstruction of the content assigned to this utterance by its utterer in the perceiver's mind. But we believe that different utterances, inscriptions, and thoughts, as well as different tokens of the same statement, thought or belief may have the same content. They cannot have this if the content does not involve some publicly accessible component. Then, there is either no common content or content cannot be exclusively mental, too.

Also, we suppose that many utterances, thoughts and beliefs may be all about the same thing or set of things. What is uttered, thought, or believed is usually supposed to be something different from how it is represented (described) in the utterance, thought or belief. The latter is normally assumed as the function of content expressed by an utterance,

And by understanding we can mean a process as well.

Relative to personal minds. thought or belief. Thus, "I am tired" says about some certain person that he/she is tired and, if true, states the fact that a certain person is tired, but what it expresses is the proposition that the author of the phrase (whoever he/she is) is tired. ${ }^{4}$ Things which words, utterances, and etc. are about are usually called denotations or references (or extensions), and they are often thought to be that what contents of meaningful expressions, utterances, and etc. should determine, which means for a particular content that it must provide enough information to make the denotation of the bearer of that content identifiable by the one who understands this content. ${ }^{5}$

Different kinds of things may figure as denotations, but there is a wide consensus among philosophers of language, linguists and psychologists that the domain of denotations of the expressions of natural languages and utterances, thoughts, etc. which use them must include real things existing independently on what we think, believe or talk. There are persons and other individual things that are supposed to exist, as well as events, places, times, properties, relations, facts, and so on. All these we may denote by using words. Thus, "I am tired" is normally supposed to expresses truth only if there is in reality, the world the interpreter lives in, someone who is really tired. ${ }^{6}$

\section{PROBLEMS WITH UNDERSTANDING OF DENOTATION}

But the alleged existence of at least two levels of meaning makes our use of the word "understanding" ambiguous; for we can say that an utterance is understood or properly understood if only its content is grasped, or that this effect may be achieved only if the right denotation is assigned. Since it is possible, in theory at least, to assign right denotation to the utterance or belief ${ }^{7}$ without assessing its content understanding of the denotation may not imply understanding of content (unless we identify content with some kind of denotation). Thus, according to J. S. Mill proper names don't have content, only denotations [4]. It may be objected that expressions used in natural languages as proper names have some content, even if only metalinguistic [5]. Either way, it may be said that it is normal for such terms that their contents (even if they have them) cannot make those who assess them understand what their denotations are. Indexical terms (like pronouns) are supposed to have yet less informative contents (if any); still, they can denote particular things, individuals, places, and time when used in appropriate context.

Some philosophers claim that there are also two levels of content, one of which is fully internal to the mind and is

\footnotetext{
On the difference between facts and propositions see: [2]. There are different notions of both proposition and fact though.

The detailed philosophical explication of this theory of meaning was first given in [3], and since then it almost dominates the philosophical and linguistic explanations of the nature of meaning.

It is not the only interpretation available though: if said by the personage of a movie or a novel this phrase would be most likely understood as true if only certain fictive person is tired in the movie or the novel.

E.g., such that is the same as the one the utterer or believer assigns to his/her utterance or belief
} 
called "narrow", whereas another is part of the world the utterance or thought is about and is called "wide". The latter should somehow combine some features of content with those of denotation. The intention behind this idea is to explain how proper names and other singular terms can contribute to the contents of utterances and thoughts which contain them, given they mean just what they denote. Thus, if the only contribution of the term "Earth" in (1) "Earth is round" to the meaning of the whole is its denotation which is usually supposed to be Earth itself, then this meaning must be denotation too. The denotation of a sentence, according to Frege, is its truth-meaning; still, we suppose that (1) expresses the proposition that Earth is round which is its content. Therefore, "Earth" must somehow contribute to the content of (2), too [6]. The only thing which this may do, according to the theory in question, is its denotation again, now treated as wide content. In this case, the very proposition which (1) expresses becomes partly something external to the mind. Now, it becomes unclear what could we mean saying that such proposition is expressed by words or contained in them. It becomes unclear how can the human mind use such propositions in understanding, interpretation and thinking, for all this requires some mental access to them. Propositions must be something which we may have in our minds if they are true contents. Any reduction of semantic contribution of linguistic expressions of a type $X$ to communication to their denotations seems to lead to the same confusion. In this case, it becomes really puzzling how anyone so limited in cognitive powers as ourselves can assign such meanings to tokens of $\mathrm{X}$, given that meaningassignment is essentially a mental act, and what it may literally use should be mental.

In model-theoretic semantics, there are claimed to be assignment-functions which link contents with denotations relative to contexts. Given the content and context, we can understand which entity in the world the utterance or the thought is about an expression denotes. But what does it mean to understand what in the world an expression denotes? This may mean different things actually. Thus, the interpreter of the utterance may understand, given the content and the context, what type of thing in the world the denotation is, i.e. how to single it out, by which features accessible in that world. This kind of understanding may be accomplished by finding and grasping the proposition which provides the best or helpful enough description of the essence or identifying properties of the denotation relative to the world. This sort of understanding barely requires more than understanding the content normally requires. But if by understanding the denotation should mean the act in which a particular entity in the world is singled out in such a way which would make it possible to use this entity instead of some content in further interpretation, thinking etc., then it seems very doubtful that normal human beings are capable of achieving results of this kind, especially in cases when the entity to be singled out is not of the sort which may be given in experience.

Are there terms which only meanings are their references or which references don't depend on their content? Is there content wide? Can we really mean them in communication?
These questions are still intensively discussed by contemporary philosophers of language. Even if we drop the idea that people are able to understand utterances and thoughts with singular terms, at least in that sense of understanding which requires singling out their referents, we still need to explain how we can understand words with other types of meanings. How can we understand contents?

\section{ThE WAy SENSE Is UNDERSTOOD}

Assessing content seems to play a key role in human understanding. What kind of thing is content? The explanation of human ability to assess contents depends on how we answer that question. If we stick to the notion of narrow content which is always "in the head" of an agent of an utterance or thought, we should treat it as something essentially mental and subjective. On the other hand, the dominant way of talking about contents is such that they are supposed to involve some intersubjective component, something due to which different tokens may have the same content. This assessable stuff is usually called sense, and it is normally claimed to belong to the domain of abstractions. As all abstractions, senses are philosophically disturbing entities, since there is no single explanation of how abstractions exist in the world which we supposed to be real. When some theory reduces them to something else which is presumed to have more definite type of existence, the price is losing some function traditionally associated with being an abstraction. Thus, the nominalist about sense may claim that "the sense of x", where x stands for an expression or any other bearer of a sense, refers to nothing but a group of particular mental contents each of which is assigned to the token of $\mathrm{x}$ in certain mental act. If so, the understanding of sense must be reduced to some sort of equilibrium between individual assignments of particular contents (which need not have common semantic features), such that being in that equilibrium provides some satisfying degree of coordination on the level of personal actions. In simple words, to understand sense in this sense is to assign such content which wouldn't prevent those who assign them effectively interact with each other and with their environment.

In this case, nothing guarantees sameness or even similarity of meanings. No two or more thoughts, utterances or beliefs would have common contents within the conceptual framework. No two tokens could be synonyms, and no two persons could say the same thing. The nominalist may insist that this is the price we have to pay if we want our theory of meaning be realistic. Still, it may be objected that, although we may hypothesize that things might be this way, we have no proof that they are, and, hence, no good reason to substitute the traditional account of sense with the reductionist one.

Can understanding be explained by reference to senses unreduced to mental or physical things? In order to be assessed, a sense must be assessable by our minds, which means that mental effects normally caused by our acquaintances with senses must represent them to us by picturing their essential or main features. Nothing guarantees that what one assigns as the same sense in different mental acts of understanding are mental representations of the same 
abstract thing, rather than different contents perceived as similar by the one who assigns them. Yet, what's less guaranteed is that the content provides the same information as content in which other people use in the same cases. It may be observed that the same skepticism may be applied to perception in general. Yet, it does not stop us from believing that we may know (although inexactly) from well refined experience how things in the world are. Why should we care about hypothetical obscurity of senses? The problem is that we may verify our empirical claims by practically testing them, whereas we cannot do the same for our claims about senses. If I see that there is a table in front of me, I may doubt that it really is there, but I may try to behave as if it is and see what will happen. Would reality support this belief or not? However, we cannot verify our judgments about senses even in this weak sense. For what should we look at in order to verify the hypotheses that certain sense was assigned? We can look at how other participants in communication react on the assignment I made (more specifically, on the action grounded in that assignment). Even if they all react in the way which doesn't disprove my assumption and even agrees with it, this would hardly tell me that I assigned the same sense as they did or would do in the same case. This set of reactions would only prove that my assignment was right in a sense of being a practical mean of coordination. If I think that I see a table this belief may be proved to be wrong by the closer look. What may work as a closer look at the sense? If, for example, I am told that Aristotle is the last great Greek philosopher, and I believe this. I will assign to the name "Aristotle" the content of the description "the last great Greek philosopher" as its sense. We suppose now that this content itself represents some abstract sense, most likely composed from senses of meaningful expression used in the description. But these senses I also assess by representing them in my mind. I can look closer at the sense of "Aristotle" by getting access to more and more detailed descriptions and other representations. They would verify my content of "Aristotle" as the representation of a certain sense only if the contents I assign to them are themselves good representations of certain senses. I have no other way to check this out than by using new contents, and their connection to any abstract sense could be questioned in the same way. There seems to be no way out of this regress comparable with what is available to us relative to the task of justification of ordinary beliefs about observable things.

\section{CONCLUSION}

W.V.O. Quine has shown that references of words used in utterances are inscrutable even when we observe what these words were applied to because what we observe is just what relevant fragments the world of utterance has but not which of them were referred to [7]. It looks like an understanding of senses as abstractions give rise to a similar problem: we cannot infer what publicly assessable senses our words used in communication have from the facts of the matter. If senses are reduced to mental or physical things (such as neural states), they seem to be incapable to make us literally "samesayers" or "samethinkers", expressing synonyms, and understanding anything such as assessing the thought expressed by someone else's use of words. Should we then drop this old-fashioned idea of understanding and substitute it with a different one that better fits the reductionist model of sense? That would depend on whether we get out with better theory then. We may define what better theory is in different ways. One of them is in terms of how many problems it solves in comparison with its rivals, and it may be claimed that a reductionist theory provides better explanation of sense because it makes senses at least determinable by certain relatively observable features. Mental contents are not directly observable of course, and it may be said that they are not quite determinable due to the very way we "observe" them. It may be answered that they are less indeterminable in that case than if realism about senses was accepted. Yet, it may be objected that this slight improvement in determinability (and it may happen to be a very small one) does not make this account preferable over the realist one which at least does not require us to break up with our familiar concepts of synonymy, generality, commonness and alike. Therefore, it seems to be no good theory of sense so far which could provide us with efficient theory of understanding. Most of the work is yet to be done.

\section{REFERENCES}

[1] D. Davidson, 'Radical Interpretation' (1973), Inquiries into Truth and Interpretation, Second Edition, Clarendon Press, Oxford, 2001, 125140.

[2] B. Russell, The Philosophy of Logical Atomism, D. Pears (ed.), Russell's Logical Atomism, London, 1972, §1.

[3] G. Frege, On Sense and Reference, Translations from the Philosophical Writings of Gottlob Frege, ed. P.T. Geach and M. Black, Oxford and New York: Blackwell, 3rd edn, 1980.

[4] D. S. Mill, A System of Logic, Volume I, London: Parker, Son, and Bourn, 1862, pp. 1, 2, 5, 33.

[5] J. J. Katz, Has the Description Theory of Names been Refuted?, Meaning and Method, ed. G. Boolos, Cambridge: Cambridge University Press, 1990, pp. 31-61.

[6] G. Frege, On Sense and Reference.

[7] W. V. O. Quine, Word and Object, MIT, 1960, pp. 28-30. 